Revue Française de Civilisation Britannique

\title{
Comparaison de la situation du chômage en France et au Royaume-Uni de 1990 à 2000
}

Joëlle Harel

\section{CpenEdition}

\section{Journals}

Édition électronique

URL : http://journals.openedition.org/rfcb/3375

DOI : $10.4000 /$ rfcb.3375

ISSN : 2429-4373

Éditeur

CRECIB - Centre de recherche et d'études en civilisation britannique

Édition imprimée

Date de publication : 1 avril 2003

ISBN : 2-911580-16-8

ISSN : 0248-9015

Référence électronique

Joëlle Harel, «Comparaison de la situation du chômage en France et au Royaume-Uni de 1990 à $2000 »$, Revue Française de Civilisation Britannique [En ligne], XII-2 | 2003, mis en ligne le 01 avril 2003, consulté le 10 décembre 2020. URL : http://journals.openedition.org/rfcb/3375; DOI : https://doi.org/ $10.4000 /$ rfcb.3375

Ce document a été généré automatiquement le 10 décembre 2020.

\section{$(1) \Theta \Theta$}

Revue française de civilisation britannique est mis à disposition selon les termes de la licence Creative Commons Attribution - Pas d'Utilisation Commerciale - Pas de Modification 4.0 International. 


\title{
Comparaison de la situation du chômage en France et au Royaume- Uni de 1990 à 2000
}

\author{
Joëlle Harel
}

1 La France et le Royaume-Uni occupent respectivement le quatrième et le cinquième rang mondial et contribuent largement à la prospérité générale. Ils font partie d'un vaste ensemble, l'Union Européenne, qui rassemble quinze pays, compte 377 millions d'habitants et dont le PNB était de 7860 milliards d'euros en $2001^{1}$. Pourtant ces deux grands pays possèdent leur part d'ombre, comme en témoigne l'existence d'un chômage de masse depuis les années soixante-dix. C'est l'aspect négatif de l'économie de ces deux grandes puissances économiques que nous allons étudier ici. Les citoyens s'inquiètent de ce phénomène qu'aucune politique ne semble capable actuellement d'éradiquer : en effet, les chiffres officiels du chômage, qui étaient de 8,9\% pour la France et de 6,9\% pour le Royaume-Uni en $1990^{2}$, restent proches de ces chiffres, puisqu'ils atteignaient en décembre 2001 9\% pour la France ${ }^{3}$, avec néanmoins une baisse de 938000 chômeurs depuis $1997^{4}$, et 5,6\% pour le Royaume-Uni ${ }^{5}$; par comparaison les États-Unis annonçaient un taux de chômage de 5,8\% en décembre $2001^{6}$ et le Japon de 5,5\%, selon The Economist du 5 janvier 2002. La France reste proche de la moyenne de l'Union Européenne, qui est de $8 \%$, mais montre son incapacité à rejoindre le groupe des pays anglo-saxons.

2 La France et le Royaume-Uni présentent donc des évolutions ${ }^{7}$ différentes dans le temps. Après avoir brièvement mentionné les situations économiques pré-existant à la période 1990-2000, nous nous proposons d'étudier les causes du chômage, les traitements sociaux mis en place par les gouvernements et enfin la réalité vécue par les chômeurs en montrant des cas pratiques afin de mieux comparer les régimes britannique et français. Une observation particulière sera portée à l'année $1995^{\circ}$ dans cet exposé, car c'est le moment où le chômage en France dépassa largement les dix pour cent, alors que la Grande-Bretagne était parvenue à maîtriser cette montée de l'inactivité pour repasser sous cette barre fatidique, en 1990. 


\section{Les causes du chômage}

3 La récession économique qui affligea les économies développées fit largement monter les taux de chômage. Ce mouvement fut renforcé par les mutations des catégories d'emplois demandés par les entreprises. La crise du chômage est le résultat d'une série de causes aux composantes multiples : évolution démographique, augmentation du taux d'activité, mutations brutales des modes de production et de gestion des entreprises, effondrement de l'industrie lourde. En effet, si la désindustrialisation fit disparaitre deux millions et demi d'emplois dans les mines et les aciéries en France et deux millions en GrandeBretagne, l'informatisation des services et l'automatisation des activités industrielles modifièrent en profondeur les besoins des entreprises. La société ne pouvait plus offrir de postes peu qualifiés car, de plus en plus, les chefs d'entreprises s'interrogèrent sur la valeur qu'ajoutaient les cols bleus à la production'. Le gain de productivité dû aux nouveaux robots convainquit les patrons de la nécessité de réorganiser les départements, peu à peu au cours des années quatre-vingt-dix. Les services eux-mêmes participèrent à ce mouvement de dégraissage et de restructuration: un grand nombre d'emplois fut remplacé par des machines automatiques ${ }^{10}$, l'organigramme des firmes fut modifié considérablement à cause de la suppression massive de l'encadrement moyen. Ainsi la diversification des tâches des employés permit-elle de limiter à quelques échelons seulement la hiérarchie des sociétés de services comme les banques ou les compagnies d'assurance ${ }^{11}$. Ces mouvements entraînèrent l'interruption provisoire, en France comme en Angleterre, du mécanisme de déversement d'un secteur économique à un autre ${ }^{12}$. Il est vrai que la Grande-Bretagne réussit à créer trois fois plus d'emplois dans le secteur marchand que la France entre 1975 et 1995.

\section{Conséquences des chocs pétroliers}

4 Le retour du chômage de masse, que les trente glorieuses avaient chassé des préoccupations quotidiennes des agents économiques, coïncida avec les deux chocs pétroliers, de 1973 et 1978, pour les pays consommateurs de pétrole comme la France ; au contraire la Grande-Bretagne devint, à la fin des années quatre-vingt, un pays producteur de pétrole en mer du Nord, ce qui bouleversa profondément sa politique monétaire.

5 La première détérioration du coût de l'énergie en 1973 avait fait croire à une crise conjoncturelle qu'une relance de type keynésien pourrait facilement endiguer, mais le second choc pétrolier de 1978 fit rechuter les indicateurs économiques de la France. Le Royaume-Uni fut lui aussi victime d'une crise économique due à une forte inflation alimentée par le statut de pétro-devise attribué à la livre sterling, à cause de la production britannique du pétrole de la Mer du Nord: le Brent. Le cours des principaux pétroles: le Brent, l'Arabian Light d'Arabie Saoudite, le Texas des États-Unis et plus récemment les types de pétrole produits par la Russie font désormais partie des indicateurs économiques que surveillent quotidiennement les économistes, les hommes politiques et les hommes d'affaires. De plus, le gouvernement de M. Thatcher, arrivé en 1979 au pouvoir, releva fortement les salaires, en particulier ceux des hauts fonctionnaires, et augmenta la TVA, aggravant ainsi la perte de compétitivité des produits britanniques sur les marchés extérieurs ${ }^{13}$. Une récession s'ensuivit, qui provoqua des faillites et une désinflation qui toucha les entreprises comme les ménages avec la 
brutale accélération du chômage ${ }^{14}$. Si l'on observe la courbe des taux de chômage français et britannique sur les trente dernières années, on s'aperçoit d'un décalage entre les deux : en effet le grand bond en avant de l'emploi se situa, au Royaume-Uni, au cours des années quatre-vingt, qui avaient pourtant commencé par une forte baisse due à la contraction de près d'un million trois cent mille emplois industriels en trois ans ; puis vint la création d'un million et demi d'emplois ${ }^{15}$, vite compensée par l'augmentation de la population active : 1,9\% par an de 1975 à 1995. C'est pourquoi le taux de chômage britannique resta stable entre 1980 à $6,1 \%$ et 1990 à 5,9\% malgré de fortes amplitudes entre ces deux dates.

\section{La désinflation compétitive, Tokyo 1979}

6 La Grande-Bretagne souffrit d'une inflation interne due au renchérissement des prix du pétrole, tandis que la France subissait une inflation importée à cause de la dégradation des termes de l'échange. Les origines opposées de ces déséquilibres n'en appelèrent pas moins des politiques monétaires harmonisées car les conséquences négatives sur la balance des paiements pénalisaient durement nos économies. En outre, l'accroissement des taux d'intérêt inhérent à cette forte inflation, la forte concurrence des produits étrangers et la hausse des matières premières, aggravèrent les déficits extérieurs, firent disparaitre les marges bénéficiaires des entreprises et multiplièrent les faillites. Tout ceci convainquit les dirigeants occidentaux qu'une politique concertée devait être entreprise pour restaurer les profits, lutter contre l'érosion monétaire et ramener la rentabilité. Il fallait agir sur l'offre et non plus sur la demande. L'année 1979 fut donc une année charnière, car elle vit le renversement de la tendance en France et en Grande-Bretagne ; de plus, le G5 réuni à Tokyo décida de suivre une politique de désinflation compétitive dans l'espoir de redresser les bilans des entreprises et le cours des monnaies.

Chaque économie s'efforça donc de reprendre des parts de marché à ses concurrents afin de retrouver la prospérité. Les entreprises limitèrent leurs investissements, leurs stocks, et licencièrent massivement leurs personnels pour réduire les coûts salariaux. Cette politique réussit dans un premier temps en Grande-Bretagne: les années 1981 à 1986 virent un rebond de la demande des ménages grâce au pouvoir d'achat dégagé par la désinflation, par une moindre pression fiscale, et par la baisse des taux d'intérêt. Le gouvernement procéda aussi à la dérégulation financière, qui favorisa le recours au crédit. Mais la situation en fin de période devint si favorable qu'elle facilita, en fait, le retour de la surchauffe de 1986 à 1988 et donc celui de l'inflation à la mi-88. Ceci amena les autorités monétaires du pays à relever fortement les taux directeurs, provoquant une forte récession en 1990. De nouveau, les entreprises durent pratiquer des déstockages massifs et des licenciements importants. Les ménages, très endettés, ne purent continuer à soutenir la demande intérieure ${ }^{16}$. Le chômage britannique passa de 7,2\% en 1979 à 10,3\% en 1986 pour repasser à 6,9\% en 1990 .

8 En ce qui concerne la France, la situation fut très différente. De plus les dirigeants étaient divisés sur l'analyse du chômage et des solutions à y apporter : la France choisit, dans un premier temps, de se rallier à la politique d'austérité telle qu'elle avait été préconisée à Tokyo. Puis, devant le manque de résultats positifs du début de la décennie, en 1981-82, elle opta pour une relance de type keynésien ${ }^{17}$, à la faveur d'un changement de gouvernement. Pourtant en 1983, trois dévaluations plus tard, le gouvernement se résigna à se rallier, de nouveau, à la politique d'austérité générale, afin de juguler l'inflation, qui redevint la préoccupation principale du gouvernement français. Il lança un 
emprunt obligatoire et surveilla les prix et les salaires. En 1986, il libéralisa le marché du travail ${ }^{18}$ en permettant aux entreprises de plus en plus de recourir aux contrats de travail temporaires, CDD, intérim, temps partiel.... Le succès couronna ses efforts car le ralentissement de la croissance qui s'ensuivit favorisa la fermeté du franc et l'inflation fut ramenée de $9,6 \%$ en 1983 à 5,8\% en 1985 et 2,7\% en 1986, ce qui était bien supérieur aux résultats des autres pays. Mais ceci fut obtenu au prix d'une forte augmentation du chômage. Les entreprises se désendettèrent au détriment des investissements, tandis que le chômage bondissait de 6\% en 1979 à 10,5\% en 1987 pour rebaisser à 8,9\% en 1990. Étaitce dû à un manque de création d'emplois ou à un trop fort accroissement de la population active?

\section{La population active}

En France, la population active continua à croître, passant de 22,2 millions à 25,5 millions de personnes de 1975 à 1995, c'est-à-dire seulement de 0,5\% par an. Il est donc erroné de dire que le chômage serait dû à une trop forte progression des actifs. Les causes sont plutôt à rechercher dans la structure du marché du travail et dans la poursuite de gains de pouvoir d'achat dans les années $1970^{19}$. Les licenciements collectifs se firent particulièrement nombreux lors de la baisse des effectifs qui se produisit d'une part de 1981 à $1986^{20}$, puis de 1991 à $1995^{21}$. En outre, au cours de la période 1989 et 1998, la population inactive de plus de quinze ans augmenta de plus d'un million deux cent mille personnes $^{22}$. L'accroissement démographique ${ }^{23}$ de la population active explique partiellement l'écart entre les taux de chômages français et britannique. Ainsi de 1975 à 1980 plus de 200000 personnes arrivèrent-elles sur le marché du travail en France, puis à partir de 1991, 150000 personnes environ.

En Grande-Bretagne, au contraire, nous avons assisté à une décrue de la population active qui se produisait depuis le début de la décennie. Elle fit baisser le taux d'activité, qui était de 75\% en 1992, à 73,9\% en 1995 à cause des diverses réformes d'inscriptions sur les listes de chômeurs : sur les exigences de mobilité géographique et de disponibilité immédiate et sur le degré d'employabilité des demandeurs d'emplois. Au Royaume-Uni, l'emploi resta à peu près constant depuis le début des années 1990, soit 27,3 millions en 1988 contre 27,1 millions en 1998. Si le taux demeura le même, c'est grâce à une modification en profondeur de la population active, qui ne progressait plus que de $0,4 \%$ contre $0,68 \%$ au cours de la décennie précédente, en raison de la baisse du taux d'activité.

\section{Politiques gouvernementales de lutte contre le chômage}

11 Les changements profonds qui transformèrent l'économie des pays développés modifièrent grandement le niveau d'employabilité des travailleurs âgés et des jeunes sans diplômes qui, jusqu'ici, parvenaient à trouver des postes n'exigeant pas de qualifications ${ }^{24}$ . Les gouvernements britannique puis français s'engagèrent dans cette bataille car ils comprirent que les entreprises, pour être performantes, avaient besoin de personnels de plus en plus qualifiés ; or naturellement la majeure partie des cols bleus mis à l'écart du marché du travail ne put s'adapter aux nouvelles exigences des sociétés, de même que la grande masse des jeunes ayant quitté l'école sans formation professionnelle ni 
intellectuelle. Aussi les politiques de l'emploi tentèrent-elles de répondre à ce défi économique et social de première grandeur.

Les entreprises eurent également une part de responsabilité dans le manque de qualification des travailleurs manuels, car elles se soucièrent peu d'offrir des stages correspondants à leurs nouveaux besoins et préférèrent engager des personnes nouvellement diplômées, immédiatement opérationnelles. En outre, la situation du chômeur favorise peu la mise à jour des compétences professionnelles, même s'il suit des stages $^{25}$ car de nombreuses formations ne sont que des 'stages-parking' et ne correspondent pas à ses attentes ou aux demandes du marché de l'emploi. Par conséquent, des effets de substitution apparurent qui permirent aux entreprises d'engager telle catégorie au détriment d'une autre, souvent avec le recours à des aides gouvernementales, sans que le chiffre global du chômage varie véritablement. Les entreprises profitèrent également d'effets d'aubaine, correspondant à des embauches qui auraient été faites de toute manière, mais qui purent être comptabilisées dans les plans gouvernementaux ${ }^{26}$ de soutien à l'emploi ${ }^{27}$, lors de recrutement de jeunes ou de chômeurs de longue durée.

13 L'existence d'un fort chômage durable a d'autres conséquences indirectes : ainsi fait-il baisser l'offre globale de travail, puisque des travailleurs se découragent ${ }^{28}$ à cause du manque d'adéquation entre leurs qualifications ou leur âge et les demandes du marché du travail. Ils disparaissent des statistiques officielles sans que la situation s'améliore pour autant.

\section{Du diagnostic au remède préconisé}

Les dirigeants politiques firent appel à de nombreux experts pour pouvoir mieux cerner le phénomène. Certains penchèrent pour un chômage de type keynésien, qui exige une relance de la demande intérieure, d'autres pensèrent avoir mis en évidence un chômage de type classique, qui réclame des baisses de salaires ${ }^{29}$ et des diminutions de prestations sociales afin de contraindre les récalcitrants à accepter de faibles salaires ${ }^{30}$. La difficulté pour les économies modernes ouvertes aux exportations des pays à bas salaires est d'harmoniser des niveaux de vie que la population ouvrière ou employée espère, puisqu'elle appartient au groupe des cinq économies les plus riches du monde, avec le niveau de revenus distribués aux travailleurs concurrents, par exemple, en Asie du SudEst.

15 Ricardo ${ }^{31}$ parlait d'un niveau de subsistance à offrir aux ouvriers anglais, mais il n'est pas certain que la population britannique ou française accepte aujourd'hui volontiers cette proposition; en outre, comment situer le niveau de subsistance en Angleterre, si on le compare avec celui des ouvriers philippins ou chinois? Les délocalisations, en favorisant l'extension des productions globales de certaines entreprises, ont déstabilisé l'équilibre précaire des pyramides des salaires dans les pays développés ${ }^{32}$. J. Plassard déclara : «Si $20 \%$ des Français sont aussi peu qualifiés que les Coréens ou les Philippins, il n'y a aucune raison de les payer plus. Il faut supprimer ce SMIC $»^{33}$.

16 Les économistes partisans de la théorie de l'équilibre général, Arrow et Debreu ${ }^{34}$ pensèrent que

les ressources initiales des agents sont suffisantes pour qu'en toutes circonstances, ils disposent d'un revenu nécessaire pour subsister pendant leur vie entière. 
Autrement dit, ils disposeraient à tout moment de l'option de ne pas travailler, option qu'ils exerceraient si le prix du travail leur semblait trop bas, ce qui garantit que le prix du travail ne baissera pas en dessous d'un certain seuil (...) d'une richesse minimum... (Mais) cette condition... n'est jamais satisfaite notamment dans nos économies, où nombre d'agents, n'ont en fait d'héritage que leur capacité de travail,

comme le déplore J. P. Fitoussi ${ }^{35}$, car la protection sociale est à ses yeux indispensable à la cohésion sociale. La persistance de ce fléau économique exige des solutions pour le bienêtre de nos sociétés et de nos entreprises. Il est en effet dommageable pour nos économies, ainsi que le rappelle l'économiste dans Le Débat interdit, qu'une partie importante de la population en âge de travailler, et désirant le faire, ne puisse contribuer à la production nationale, et que tous les accroissements de productivité dont nos économies sont capables ne puissent être exploités pour renforcer encore davantage notre prospérité ${ }^{36}$. Car le véritable test d'une économie, mesurée par de nombreux indices, est le niveau de vie de ses agents et le volume de son PNB, souligne cet auteur.

\section{Réduction des chiffres du chômage}

171905 vit la Grande-Bretagne, avec The Unemployed Workmen Act, et la France, avec La Charte de l'Assurance Française contre le Chômage, reconnaître l'existence du chômage involontaire et le statut de victime aux sans-emploi, jusqu'ici considérés comme seuls responsables de leur situation ${ }^{37}$. A partir de ce moment-là, les gouvernements durent faire face aux demandes d'aide des sans-emploi selon la conjoncture. Cependant, les dirigeants politiques voulurent de nouveau dans les années 1990, limiter les dépenses 'passives' d'aide aux chômeurs pour renforcer les politiques de l'emploi ou classer les demandeurs d'emploi dans des catégories différentes ${ }^{38}$ afin de concentrer leur soutien financier sur les demandeurs d'emploi qui avaient véritablement une chance de retrouver un travail rapidement.

Les gouvernements britannique et français cherchèrent tout d'abord à réduire par tous les moyens le taux du chômage qui les obsède, car il témoigne d'un malaise profond de la société et d'une incapacité des dirigeants à résoudre une des tâches pour lesquelles ils ont été portés au pouvoir ${ }^{39}$.

19 Aux vagues successives de licenciements des années quatre-vingt, succédèrent des plans de restructuration moins brutaux incluant le recours au sous-emploi et aux préretraites. Ainsi selon l'OCDE, en $1993^{40}$, on comptait $14 \%$ de travailleurs en France dans le cadre du travail partiel. On constate la progression de ce phénomène puisque $38,7 \%$ des postes à temps partiel ${ }^{41}$ étaient en fait des situations acceptées faute de mieux ${ }^{42}$, soit à cause du marché du travail, du manque de mobilité géographique du travailleur ou d'obligations personnelles ou familiales qui n'avaient pu être résolues. Ce qui représente pour la France, en 1995, 1,3 million d'actifs ${ }^{43}$. 12,5\% des actifs en Grande-Bretagne étaient sousemployés ${ }^{44}$, cette année-là, selon l'OCDE. Les dispositifs de préretraite, très utilisés en France dès 1983, permirent une baisse artificielle des chiffres du chômage. De plus, depuis 1997, les demandeurs d'emploi âgés de plus de cinquante-cinq ans ne sont plus obligés de rechercher un emploi et ne figurent plus dans le fichier de l'ANPE, et 30\% de ceux qui ont cinquante ans ne sont plus assujettis à cette mesure. Cette façon d'exclure du décompte officiel du chômage les travailleurs âgés est intéressante pour le gouvernement puisqu'à la fin de la décennie 1990, 55\% des chômeurs de longue durée appartenaient à cette tranche d'âge ${ }^{45}$. Des préretraites de complaisance, aussi, furent accordées aux entreprises 
en période de restructuration avec le système du contrat de solidarité qui permit à 200 000 jeunes d'obtenir un emploi à condition qu'un départ à la retraite accompagne ces embauches. Or l'État participe au paiement des préretraites. En 1996 les retraites coûtèrent 18 milliards de francs à l'UNEDIC, soit le quart des ressources ${ }^{46}$.

\section{Les régimes d'assurance et de solidarité}

Le partage des aides entre l'assurance et la solidarité est différent que l'on soit en France ou en Angleterre : ainsi sur les 100 milliards de francs dépensés par l'État français, 90\% l'étaient au titre de l'assurance chômage, le reste étant affecté à la solidarité. L'Angleterre, elle, se déclara peu encline à verser autant d'argent dans ces postes budgétaires. Pourtant, les Britanniques durent également consacrer des sommes importantes à la survie des sans-emploi, malgré les déclarations de John Major ${ }^{47}$ en faveur de la politique pour l'emploi. En France, le 21 juillet 1992 l'Allocation Unique Dégressive, l'AUD $^{48}$ fut mise en place et revit à la baisse le montant perçu ${ }^{49}$ et le volume des bénéficiaires; en effet un nombre croissant de personnes se trouvèrent exclues à cause $d u$ caractère précaire de leur emploi qui ne leur permettait pas de présenter une durée suffisante de cotisations.

En 1988, le Revenu Minimum d'Insertion fut instauré ${ }^{50}$ en France $^{51}$ et il donne droit à une aide mensuelle de $2400 \mathrm{~F}$ pour les bénéficiaires de l'Allocation de Solidarité Spécifique et à $1900 \mathrm{~F}$ pour ceux qui perçoivent l'Allocation Unique Dégressive. Le RMI est accordé aux personnes sans ressources âgées de 25 ans au moins. Il est, en fait, assimilé à un second régime d'allocation chômage puisque, en 1999, 10\% des sans-emploi le touchaient et que $70 \%$ des bénéficiaires étaient au chômage depuis plus d'un an. Si en France les allocations chômage ne sont pas liées au niveau de ressources familial, les versements au titre du RMI, au contraire, le sont: «pour déterminer le montant du RMI applicable à la famille on prend en compte non seulement le demandeur; mais également s'il réside (avec un) conjoint, concubin ou partenaire lié par un PACS $\aleph^{52}$.

Comme l'écrivent C. André et D. Sicot :

Le système britannique est très différent de celui des autres pays européens, car il n'est pas lié aux salaires antérieurement perçus mais dépend des revenus imposables perçus pendant les deux dernières années d'imposition ${ }^{53}$. En outre la «garantie de revenu » (Income Support) n'est versée que si les autres membres de la famille du chômeur n'ont pas de travail à temps plein ou si leurs revenus sont faibles..$^{54}$

L'actuelle allocation chômage en place depuis 1996, la Job Seekers' Allowance, s'accompagne d'un Job Seeker's Agreement qui contraint le signataire adulte après deux ans de chômage à accepter soit une formation soit une offre d'emploi ou d'activité d'intérêt général, même précaire ou mal payée, sous peine d'être radié des registres. Pour un jeune, la durée du chômage nécessaire est réduite à six mois. Si le demandeur d'emploi est toujours sans situation par la suite, il reçoit une aide au titre de la solidarité, l'Income Support.

24 Une politique énergique du New Labour intitulée le New Deal pour les jeunes de 18 à 24 ans chercha à convaincre les chômeurs que "le travail paie ", ainsi que l'affirma Tony Blair en lançant sa campagne, Welfare-to-Work, en 1997. Il renforça les critères de plafonds de ressources et de recherche active d'un emploi. La France tenta, plus récemment, d'appliquer la même politique avec le Plan d'Aide au Retour à l'Emploi, PARE, qui est officiellement facultatif pour les chômeurs de longue durée, inscrits avant sa mise en 
application en 2001, mais devint obligatoire pour les chômeurs inscrits après le $1^{\text {er juillet }}$ $2001^{55}$. En 1998, les chômeurs de longue durée représentaient $40 \%$ du total au RoyaumeUni et $45 \%$ du total des chômeurs en France ${ }^{56}$.

La réintroduction par le gouvernement de Margaret Thatcher des plafonds de ressources, les Means Tests, suppose qu'une enquête financière soit menée par les services prestataires. La famille est, désormais, considérée dans son ensemble : s'y trouvent inclus les partenaires et les enfants des demandeurs d'allocations. Des questions précises tentent d'évaluer le degré d'intimité qui existent entre certains adultes de manière à comptabiliser les concubins éventuels dans la famille. En effet, en 1995 selon le Central Statistical Office, $25 \%$ seulement des ménages étaient composés d'un couple marié et d'enfants à charge, à cause de l'augmentation du nombre de célibataires avec ou sans enfant.

Au Royaume-Uni comme en France, le montant des aides distribuées aux sans-emploi dépend de la situation économique et fluctue donc d'année en année, à cause de la longueur du phénomène et de son importance, car il est devenu primordial d'équilibrer les dépenses avec les recettes affectées officiellement à ce poste.

\section{La réalité de la vie des chômeurs}

Certes, le chômage n'est pas toujours durable et structurel comme nous l'avons vu essentiellement jusqu'à maintenant. Il peut être «frictionnel » c'est-à-dire frapper des individus en période de transition entre deux emplois pendant trois à six mois. Ce type de chômage concernait un million de personnes en France en 1997. Pourtant c'est la catégorie des chômeurs chroniques qui nous intéresse de nouveau ici, car c'est sur cette partie de la population que les effets du chômage sont les plus sensibles. A la recherche des causes du chômage, certains experts se sont interrogés sur le degré de motivation qu'éprouvaient les sans-emploi pour se mettre en quête véritablement d'un poste.

\section{Le degré de motivation de la population active}

Des études ont été menées afin de mieux cerner la personnalité des demandeurs d'emploi et pouvoir affiner les politiques de lutte contre le chômage. Les résultats concernant le taux de motivation des chômeurs et des travailleurs en France et en Angleterre sont riches d'enseignements, à cet égard.

En France, seulement 59,4\% des chômeurs et 36,9\% des actifs, contre 78,3\% des chômeurs et $53 \%$ des actifs, en Angleterre, désireraient travailler s'ils étaient assurés de jouir d'un revenu suffisant pour leur assurer une vie confortable pour le restant de leur vie. Il apparaît tout d'abord que la motivation des sans-emploi est plus forte que celle des actifs. Sans doute apprécient-ils davantage la chance de mener une vie structurée et équilibrée qui leur offre un but chaque jour, face au néant que peuvent représenter, pour certains, l'inactivité et la déstructuration sociale, les conduisant à la dépression. C'est l'hypothèse que formulèrent les auteurs ${ }^{57}$ de cette étude. En outre, on observe que les Français semblent moins enclins à exercer une activité rémunérée que les Britanniques. Peut-être sont-ils motivés par des intérêts intellectuels, culturels, sportifs, ludiques ou éducatifs devenus plus accessibles lorsque la contrainte du travail disparait ? 
30 La société, cependant, subit les conséquences induites d'un chômage de masse durable, car si les coûts directs au titre des différentes aides sont considérables, les coûts indirects se révèlent également très élevés, en particulier lorsqu'ils résultent de la criminalité. Une économie souterraine se crée, avec ses propres règles, et se développe aux dépens de l'économie officielle.

31 De plus, le faible écart qui existe entre les aides sociales et les salaires de base découragent parfois certains demandeurs d'emploi. C'est un point de vue largement développé Outre-Manche, où les autorités veillent à ne pas pousser à l'inactivité des travailleurs potentiels. Il est remarquable qu'en France un bénéficiaire du RMI, ayant deux enfants à charge, perde 10000 francs sur l'année s'il accepte un poste payé au SMIC ${ }^{58}$ - Peut-être une revalorisation considérable des salaires éviterait-elle de placer les chômeurs devant ce dilemme ? Car baisser les aides, comme cela est préconisé et effectué en Grande-Bretagne, risquerait d'enfermer ces personnes dans le piège de la pauvreté, la poverty trap des sociologues anglophones. "12 millions de Français dépendent des transferts sociaux pour assurer leur subsistance » rappela N. Baverez, en 1997, dans Les trente piteuses ${ }^{59}$.

\section{Comparaison des allocations offertes aux chômeurs français et britanniques}

Il reste difficile de comparer terme à terme les politiques sociales des deux pays, qui ne suivent pas les mêmes principes : si la durée des prestations est beaucoup plus courte en Angleterre, le pourcentage des bénéficiaires est plus élevé, sans doute à cause du basculement des sans ressources vers le RMI en France. En 1995, 59,4\% des chômeurs recevaient une allocation, contre $45 \%$ seulement en France, mais les bénéficiaires britanniques touchaient une allocation moindre que les Français ${ }^{60}$. Voici sans doute pourquoi il est nécessaire de quitter la macro-économie pour observer de plus près les conséquences des politiques gouvernementales sur la vie des chômeurs et de comparer les résultats chiffrés à l'aide de quatre cas particuliers, illustrant des exemples précis ${ }^{61}$, parus dans deux revues spécialisées en 1998. Tous les salaires cités sont des rémunérations brutes.

33 Ainsi Liaisons Sociales publia-t-elle, le 7 août 1998, une étude comparative afin de mieux faire comprendre le quotidien des chômeurs en France et en Grande-Bretagne. Le premier cas concernait un demandeur d'emploi de moins de cinquante ans avec un revenu mensuel de 8000 francs et n'ayant cotisé que pendant la durée minimale autorisée.

\begin{tabular}{|l|l|l|l|l|l|}
\hline Pays & $\begin{array}{l}\text { Durée de } \\
\text { cotisation }\end{array}$ & $\begin{array}{l}\text { Montant de deindemnisation } \\
\text { l'indemnisation }\end{array}$ & $\begin{array}{l}\text { Durée } \\
\text { l'issistance } \\
\text { en fin de } \\
\text { droits }\end{array}$ & Observations \\
\hline France & $\begin{array}{l}4 \text { mois } \\
\text { sur } 8\end{array}$ & $5020 \mathrm{~F} /$ mois & 4 mois & $\begin{array}{l}2400 \text { F par } \\
\text { mois selon } \\
\text { ressources }\end{array}$ & $\begin{array}{l}\text { Ouverture de } \\
\text { droits au bout de } \\
\text { mois d'activité }\end{array}$ \\
\hline $\begin{array}{l}\text { Grande- } \\
\text { Bretagne }\end{array}$ & $\begin{array}{l}\text { Plafond de } \\
\text { cotisations }\end{array}$ & $1990 \mathrm{~F} /$ mois & 6 mois & $\begin{array}{l}\text { Allocation } \\
\text { d'assistance } \\
\text { selon } \\
\text { ressources }\end{array}$ & $\begin{array}{l}\text { Rendez-vous au } \\
\text { Job Centre tous les } \\
15 \text { jours, pour } \\
\text { avoir l'allocation }\end{array}$ \\
\hline
\end{tabular}


Une autre étude de cas, publiée cette fois par l'UNEDIC, proposait d'observer trois exemples. D'abord, le cas d'un homme de quarante ans, ayant travaillé pendant quinze ans, marié père de deux enfants, et gagnant 15000 francs par mois tandis que sa femme touchait 8000 francs mensuellement.

\begin{tabular}{|l|l|}
\hline Pays & Indemnisation \\
\hline France & $\begin{array}{l}2,5 \text { années d'allocation unique dégressive, l'AUD : } 6800 \text { F les neuf premiers mois, } \\
\text { puis }-15 \% \text { tous les } 4 \text { mois, les } 3 \text { derniers mois étant fixés à } 3200 \mathrm{~F} . \text { L'allocation de } \\
\text { solidarité spécifique, l'ASS, prend le relais }\end{array}$ \\
\hline $\begin{array}{l}\text { Royaume- } \\
\text { Uni }\end{array}$ & $2000 \mathrm{~F} /$ mois pendant 6 mois \\
\hline
\end{tabular}

L'UNEDIC ajouta un autre exemple, celui d'une femme âgée de cinquante-huit ans qui avait travaillé pendant trente-cinq ans avec une interruption de cinq ans. Elle était mariée à un retraité qui gagnait 8000 francs par mois et n'avait plus d'enfant à charge.

\begin{tabular}{|l|l|}
\hline Pays & Indemnisation \\
\hline France & $\begin{array}{l}8700 \mathrm{~F} / \text { mois pendant } 2 \text { ans et elle atteindra l'âge requis } \\
\text { pour jouir d'une retraite à taux plein }\end{array}$ \\
\hline Royaume-Uni & $2000 \mathrm{~F} /$ mois pendant 6 mois \\
\hline
\end{tabular}

Enfin l'UNEDIC mentionna l'histoire d'une jeune femme de vingt ans qui avait travaillé cinq mois durant l'année écoulée, pour un salaire de $6000 \mathrm{~F}$ mensuels.

\begin{tabular}{|l|l|}
\hline Pays & Indemnisation \\
\hline France & $4730 \mathrm{~F} /$ mois pendant 4 mois \\
\hline Royaume-Uni & $1660 \mathrm{~F} /$ mois durée illimitée, car elle touchera alors l'Income Support \\
\hline
\end{tabular}

Comme on le voit, l'ensemble des prestations britanniques est inférieur aux prestations françaises, en particulier en ce qui concerne les chômeurs âgés de plus de vingt-cinq ans. La modicité des aides distribuées aux sans-emploi les exclut partiellement de la consommation alors qu'ils sont tout autant sollicités que les autres citoyens par la publicité et la société d'abondance qui nous entoure. Ils sont donc la proie de frustrations répétées qui ne peuvent apaiser le sentiment d'inutilité et d'incapacité qui les anéantit. Les mouvements d'appel à la générosité de l'État que les chômeurs manifestent à certaines occasions témoignent de cette souffrance, car un grand nombre d'entre eux sont d'authentiques et d'honnêtes demandeurs d'emploi qui souhaiteraient pouvoir échapper à leur oisiveté forcée, retrouver leur place de producteurs de la richesse nationale et participer à la frénésie de consommation qui étreint les populations des pays 
développés, que ce soit à Noël ou au moment des vacances par exemple. C'est pourquoi les chômeurs risquent de sombrer dans la pauvreté et principalement les jeunes.

\section{Impact du chômage sur le niveau de vie}

Le chômage s'accompagne souvent de la montée des situations d'exclusion. Une étude du CREDOC, organisme français chargé de mesurer la consommation, permet de dégager trois mécanismes qui mènent à la pauvreté: les chômeurs ayant épuisé toutes les allocations et arrivant en fin de droits, l'impossibilité pour les jeunes de toucher le RMI avant l'âge de vingt-cinq ans et le basculement de la vie privée à cause d'un divorce ou d'un décès. En outre, on observe les phénomènes anciens de reproduction de la pauvreté de génération en génération qui se perpétuent par manque de formation et d'esprit d'initiative ${ }^{62}$.

Devant les angoisses et les souffrances que génère le chômage il est peu surprenant que les jeunes cherchent à fuir ce type de situation; ainsi en France une enquête, Jeunesse et Entreprise, effectuée sous la direction d'Yvon Gattaz, auprès de 45000 bacheliers, en 1996, faisait-elle état du fait que $69 \%$ des jeunes de 18 à 24 ans craignaient de tomber dans l'exclusion, peur qui n'affectait que $28 \%$ des personnes de plus de soixante-cinq ans ${ }^{63}$. De plus, $12 \%$ des jeunes voulaient un emploi en entreprise tandis que $50 \%$ d'entre eux espéraient devenir fonctionnaires. Ils souhaitaient travailler mais espéraient ne pas être placés sous l'épée de Damoclès que constitue le chômage. Il est vrai que le chômage des jeunes non diplômés passa de 9,9\% en 1993 à 20,3\% en $1997^{64}$.

De nombreux enfants adultes restent vivre chez leurs parents soit par attachement filial, soit pour aider l'un ou l'autre parent âgé à assumer les tâches quotidiennes, mais aussi par manque de revenus suffisants pour s'installer seuls. Or la cohabitation entre générations a de fortes conséquences sur le calcul des indemnités versées à la famille, en France comme en Grande-Bretagne. Cette solidarité, qui se manifeste davantage en France qu'en Grande-Bretagne, révèle donc le fort lien qui unit les membres d'une même famille.

Deux tableaux établis lors d'une enquête de la Commission Européenne ${ }^{65}$, reprise par D. Gallie et S. Paugam, illustrent la solidarité familiale et le degré de pauvreté des sansemplois :

i) Proportion d'enfants adultes en 1994 de 20 à 29 ans vivant chez leurs parents :

\begin{tabular}{|l|l|l|}
\hline Pays & Tous les Jeunes de 20 à 29 ans & Chômeurs de 20 à 29 ans \\
\hline France & $39,5 \%$ & $48,5 \%$ \\
\hline Royaume-Uni & $32,4 \%$ & $41,7 \%$ \\
\hline
\end{tabular}

ii) Difficultés financières parmi les chômeurs en $1994^{66}$ :

\begin{tabular}{|l|l|l|}
\hline Pays & Difficultés financières & Privations matérielles \\
\hline France & $41 \%$ & $4,8 \%$ \\
\hline
\end{tabular}


\begin{tabular}{|l|l|l|}
\hline Royaume-Uni & $45 \%$ & $5,1 \%$ \\
\hline
\end{tabular} chercheurs concernant le risque de tomber sous le seuil de pauvreté des personnes à risques : les personnes au chômage depuis plus de douze mois, les demandeurs d'emploi qui avaient eu des bas salaires auparavant, les chômeurs vivant seuls et enfin les mères élevant seules leurs enfants, en France comme en Grande-Bretagne.

\section{Conclusion} charges sociales patronales, fut accusé, selon le terme consacré, de dumping social envers la France, ainsi que les travailleurs français de Hoover ${ }^{67}$ s'en souviennent. Mais désormais la construction européenne, renforcée par l'arrivée de l'euro dans douze pays de l'Union pourra peut-être permettre d'harmoniser les politiques fiscales et sociales qui attirent certains chefs d'entreprises en dehors de nos frontières. Le problème de la résorption du chômage se pose en effet à l'ensemble de l'Union Européenne. En 1990, on y comptait douze millions trois cent mille chômeurs alors que ce chiffre passa à dix-sept millions cent mille en 1999, c'est-à-dire $10 \%$ de la population active. Certes, cette proportion a baissé quelque peu en 2001 pour retrouver un taux de 8\%, mais l'ampleur de la tâche devra mobiliser encore les gouvernements pendant quelques années, s'ils veulent tenir les engagements pris devant leurs électeurs au cours des diverses campagnes électorales récentes.

L'idée d'une politique sociale communautaire apparut au sommet de Paris de 1972, nous rappellent les rédacteurs de l'IRES; elle fut relancée en 1984 lors du sommet de Fontainebleau. En 1989, onze états membres adoptèrent au sommet de Strasbourg la Charte Communautaire des Droits Sociaux Fondamentaux des Travailleurs, que le Royaume-Uni signa avec le Traité d'Amsterdam en 1997. Ceci permit à l'Angleterre de se doter pour la première fois d'un code du travail. Jusqu'à présent, le contrat de travail était régi par le droit commercial commun, négociable entre employeur et employé à l'exception des règles touchant à la discrimination raciale et sexiste et au droit des enfants. Depuis la suppression des Wages Councils et avec la faiblesse accrue des syndicats, les employés avaient peu de pouvoir face à leur entreprise en matière de contrat de travail.

Malgré les vives préoccupations de la population européenne depuis des décennies, l'emploi ne fut mentionné qu'en 1993 avec le Livre blanc du ministère du Travail britannique, Croissance, Compétitivité et Emploi. Mais la construction d'un marché du travail unique est encore éloignée dans le temps, car cela nécessiterait une convergence des droits du travail, des avantages sociaux et des réglementations fiscales, ce qui n'est pas encore à l'ordre du jour. 


\section{NOTES}

1. Derrière les États-Unis avec 9102 milliards d'euros et devant le Japon avec 4381 milliards d'euros, soit pour les Européens un PNB par habitant de 22529 euros, les Américains ayant 33042 euros et les Japonais 34591 euros par habitant, étant donné leur plus faible population. Le Monde, $1^{\text {er }}$ janvier 2002, « L'arrivée de l'euro » p. 5.

2. D'après l'OCDE, Perspectives de l'emploi, juillet 2000, in IRES, 2000, p. 29.

3. Et pourtant le préambule de la constitution du 27 octobre 1946 repris en 1958 affirme que « chacun a le devoir de travailler et le droit d'obtenir un emploi... Tout être humain qui, en raison de son âge, de son état physique ou de la situation économique, se trouve dans l'incapacité de travailler a le droit d'obtenir de la collectivité des moyens convenables d'existence ».

4. Le professeur Guillaume de l'Université Paris X confirma la baisse du chômage en France et déclara que les trente-cinq heures avaient contribué à la création d'environ 200000 emplois, $L C I$, 4 janvier 2002, sur les 350000 postes mis à la disposition des services publics, le secteur privé ayant boudé ces emplois pourtant lourdement subventionnés à hauteur de $80 \%$ des salaires.

5. Le Monde du 4 décembre 2001 reprenait des chiffres publiés par Eurostat en juillet 2001, 8,6\% pour la France et $5,1 \%$ pour le Royaume-Uni, qui montrent que ce mouvement s'est accéléré depuis l'été 2001, Le Monde Emploi, p. X, 4 décembre 2001.

6. CNN, 4 janvier 2002.

7. Taux de chômage standardisé de 1973 à 2001 :

\begin{tabular}{|l|l|l|l|l|l|l|l|l|l|}
\hline Pays & $\mathbf{1 9 7 3}$ & $\mathbf{1 9 7 9}$ & $\mathbf{1 9 8 3}$ & $\mathbf{1 9 9 0}$ & $\mathbf{1 9 9 5}$ & $\mathbf{1 9 9 7}$ & $\mathbf{1 9 9 9}$ & $\mathbf{2 0 0 0}$ & $\mathbf{2 0 0 1}$ \\
\hline France & $\mathbf{2 , 7} \%$ & $5,9 \%$ & $8,3 \%$ & $8,9 \%$ & $12,5 \%$ & $12,5 \%$ & $11,3 \%$ & $9,1 \%$ & $9 \%$ \\
\hline GB & $2,2 \%$ & $5 \%$ & $12,4 \%$ & $6,9 \%$ & $8,7 \%$ & $5,8 \%$ & $\mathbf{6 , 7} \%$ & $5,5 \%$ & $5,6 \%$ \\
\hline
\end{tabular}

d'après Perspectives de l'Emploi, OCDE, juillet 1998, juillet 2000, in Le Monde, 4 janvier 2002, L'arrivée de l'euro, p. X

8. Nature du chômage en 1995 en taux standardisés :

\begin{tabular}{|l|c|c|c|}
\hline Pays & $\begin{array}{c}\text { \% chômage } \\
\text { longue durée }\end{array}$ & $\begin{array}{c}\text { \% chômage } \\
\text { des 18-24 ans }\end{array}$ & $\begin{array}{c}\text { \% chômage } \\
\text { des femmes }\end{array}$ \\
\hline France & $5,3 \%$ & $25,9 \%$ & $14,2 \%$ \\
\hline GB & $3,8 \%$ & $15,5 \%$ & $4,3 \%$ \\
\hline
\end{tabular}

d'après Perspectives de l'Emploi, OCDE juillet 1996.

9. On assista à la fin du modèle fordiste qui avait soutenu la croissance des grands pays industrialisés et ce phénomène prit une ampleur considérable tandis que les secteurs industriels américains et japonais résistèrent jusqu'à la fin du siècle.

10. La distribution utilise les robots et les lecteurs de codes-barres, la télématique évite la livraison de courrier et trouve sa place dans la gestion commerciale des firmes, les guichets automatiques suppléent à la vente de tickets..., J. RIFKIN, The End of Work, New York: Simon \& Schuster, 1995, p. 196 et seq.

11. J. RIFKIN, op.cit., 1995.

12. G. AZNAR, La fin des années chômage : La stratégie de l'emploi, Paris : La Découverte \& Syros, 1999.

13. Hervé MONET \& Jean-Jacques SANTINI, L'économie britannique : le libéralisme à l'épreuve des faits, Paris : Nathan, 1992.

14. Le mot 'chômage' vient du latin 'caumare' et du grec 'kauma' qui veut dire 'chaleur torride', c'est-à-dire période pendant laquelle il faisait trop chaud pour travailler dans les champs.

15. L'emploi féminin passe de $40 \%$ à $44 \%$ de l'emploi total, le travail à temps partiel de $18,4 \%$ à $21,7 \%$ et le travail indépendant de 8,7\% à $13 \%$ entre 1980 et 1990, IRES, 2000, p. 38.

16. Hervé MONET \& Jean Jacques SANTINI, op. cit., 1992. 
17. Le gouvernement revalorisa le SMIC, augmenta les diverses prestations sociales, créa 240000 emplois publics, fit voter la loi des 39 heures et une cinquième semaine de congés payés, abaissa l'âge de la retraite de soixante-cinq à soixante ans, établit des contrats de solidarité qui permirent d'embaucher 200000 jeunes à condition qu'autant de travailleurs âgés partent à la retraite. La croissance française était de $2 \%$ supérieure à celles de ses partenaires en 1982, mais le chômage ne fut que stabilisé. Le coût des mesures sociales fit doubler le budget des dépenses de ce poste. Le projet de baisser la durée du travail de la semaine à trente-cinq heures à l'horizon de 1986 fut abandonné. L'aggravation de l'inflation et la chute de la demande entraînèrent une hausse du déficit commercial de trente milliards de francs. Jacques BOUCHOUX \& Marc HEMELIN, A chaque État son chômage, Paris : Hatier, 1991, p. 46 et sq.

18. Le gouvernement créa les TUC, contrats de travaux d'utilité publique, semblables aux TEC britanniques, qui concernèrent 200000 jeunes, des SVIP, stages d'insertion à la vie professionnelle, pour favoriser l'intégration des jeunes ou des PIL, destinés aux chômeurs plus âgés. J. BOUCHOUX \& M. HEMELIN, op.cit., p. 51.

19. Nicolas BAVEREZ, Les Trente Piteuses, Paris : Flammarion, 1997.

20. En 1986, Yvon Gattaz, président du CNPF, obtint du gouvernement français la suppression de l'autorisation préalable de licenciement en assurant les autorités qu'un assouplissement du marché du travail permettrait au contraire l'embauche de 400000 personnes. Pourtant après une pause, le chômage continua de progresser, Olivier MAZEL, La France des chômages, Paris: Gallimard, 1999, p. 78.

21. Le secteur secondaire perdit 100000 emplois par an de 1980 à 1986, puis plus de 60000 de 1992 à 1995, et le secteur marchand n'engagea plus à ces périodes-là, N. Baverez, op. cit., 1997, p. 42 et sq.

22. IRES, 2000.

23. Le solde migratoire en France depuis 1945 est de 12 millions de personnes soit $20 \%$ de l'augmentation totale de la population active, J. Bouchoux \& M. Hemelin, op. cit., p. 42.

24. Le Monde, 24 avril 1996.

25. Les stages de formation linguistiques, par exemple, qui sont proposées par un certain nombre d'entreprises n'offrent pas les conditions d'un véritable apprentissage à raison d'une heure par semaine sur une période de trois mois comme au Crédit Lyonnais en 1982 ; parfois ces formations sont accompagnées d'une mise en demeure de devenir 'bilingue' en quelques mois sous peine de licenciement comme dans la Midland Bank à Paris en 1983, ainsi que l'auteur put le constater sur le terrain.

26. Les dépenses actives pour l'emploi en pourcentage du PIB furent de $0,7 \%$ en $1985,0,8 \%$ en 1990, 1,4\% en 1997 en France et $0,7 \%$ en 1985, 0,6\% en 1990 et 0,4\% en 1997 pour la GrandeBretagne, ce qui représenta $42,5 \%$ des dépenses totales de l'emploi en 1997, pour la France, et 31,1\%, pour le Royaume-Uni, Perspectives et emploi, OCDE, juillet 1999.

27. L'OCDE publia les taux des dépenses publiques en faveur du marché du travail et du chômage. La France dépensa $1,37 \%$ au titre des dépenses actives et $1,50 \%$ de son PIB en 1997 pour le chômage, tandis que la Grande-Bretagne dépensa $0,42 \%$ au titre de la politique active et $1,05 \%$ de son PIB pour lutter contre le chômage OCDE, Perspectives de l'emploi, juillet 1999.

28. 500000 personnes étaient considérées dans cette catégorie par l'ANPE en 1997, G. AZNAR, op.cit, 1999, p. 258.

29. Pour RICARDO, le salaire de subsistance est : « le prix naturel du travail... qui fournit aux ouvriers les moyens de subsister et de perpétuer leur espèce sans accroissement ni diminution ", O. MAZEL, La France des chômages, Paris : Gallimard, 1999, p. 81.

30. Dans une logique libérale, le chômage "serait le résultat d'un choix individuel: le demandeur d'emploi compare d'une part le coût de la recherche d'un emploi et d'autre part le bénéfice actualisé prévisible », Jacques FRESSINET, Le chômage, Paris : La Découverte, 1998, ... (pourtant) «la baisse 
régulière, ces dernières années, des conditions d'indemnisation a eu peu d'impact sur le nombre des chômeurs et sur la fluidité du marché du travail » O. MAZEL, op. cit., 1999, p. 78.

31. David RICARDO, Principles of Political Economy and Taxation, 1817 (Principes de l'Économie Politique et de l'Impôt, Paris, Calmann-Levy, 1970).

32. Comment s'étonner alors que certains chômeurs refusent les salaires compétitifs qui leur sont offerts? Les métiers intellectuels ne sont pas à l'abri. Des docteurs en informatique venus de l'Inde travaillent dans les firmes multinationales, d'autres docteurs enseignent dans les meilleures universités américaines pour quelques milliers de dollars. Des pilotes russes proposent leurs services à des compagnies occidentales pour des salaires dérisoires, très loin des revenus actuels des pilotes européens.

33. B. PERET \& G. ROUSTANG, L'Économie contre la Société, Paris : Seuil, 1983, p. 104.

34. K. ARROW \& G. DEBREU, 'Existence of an equilibrium for a competitive economy' Econometrica , 1954, n²2, pp. 45-55.

35. Jean-Paul FITOUSSI, Le Débat interdit : Monnaie, Europe, Pauvreté, Paris : Arléa, 1995, p. 119 et sq. 36. Le sénateur Jean Artuis ... " estime qu'au minimum entre trois et cinq millions d'emplois... sont menacés directement par les mouvements de délocalisations", Rapport du Sénat sur les Délocalisations, 1993, in O. MAZEL, op. cit., 1999, p. 79.

37. En France, en mai 1939 le code du chômage indique que «les chômeurs sont les travailleurs habituellement occupés par un employeur et tirant de cette occupation une rémunération régulière et non une rémunération d'appoint, lorsqu'ils ont perdu leur occupation et qu'ayant toute liberté d'en accepter une autre il ne peut leur en être procuré une autre, bien qu'ils aient la capacité et la volonté de travailler». Charles WILLIAM, L'identité juridique du chômeur, Paris, LCDJ, 1998, p. 121. En 1958, l'UNEDIC et les ASSEDIC furent créées pour indemniser les sans-emploi, suivies de l'ANPE en 1967, qui se chargea de mettre en contact les offres d'emplois et les personnes à la recherche d'une situation.

38. Depuis 1985 l'ANPE classe les demandeurs d'emploi en huit catégories différentes selon la durée de l'emploi demandé et le type d'emploi, à temps partiel ou à temps plein, et la disponibilité des personnes. En outre elle ne s'intéresse désormais qu'aux demandeurs d'emploi et non plus aux chômeurs, car l'inscription à l'ANPE n'est pas obligatoire. Les offres d'emploi sont classées en trois groupes, O. MAZEL, op. cit., 1999, p. 41 et sq. «Un demandeur d'emploi est une personne sans emploi et disponible pour en occuper un qui fait la démarche de s'inscrire ou de confirmer par courrier tous les mois son inscription à l'agence. Depuis octobre 1996... l'inscription donnant droit aux indemnisations est prise en charge par les bureaux locaux des ASSEDIC; l'ANPE n'assurant plus que la fonction d'accompagnement du demandeur d'emploi dans sa recherche d'emploi», O. MAZEL, op. cit., 1999, p.42.

39. Les marchés du travail en Europe, IRES, Paris : La Découverte, 2000, p. 65.

40. Par comparaison le taux de chômage en France était de 11,4\% et celui de la Grande-Bretagne de 10,3\% la même année, en taux standardisés, Perspectives de l'Emploi, OCDE, juillet 1995.

41. Les taux de temps partiel involontaire étaient de $0,2 \%$ pour la France et de 0,6\% pour la Grande-Bretagne en 1993, OCDE, Perspectives pour l'Emploi, juillet 1995.

42. L'INSEE publia un tableau montrant l'évolution du sous-emploi en France de 1992 à 1996 concernant les travailleurs à temps partiel cherchant un emploi à temps complet ou plus long en :

$1992: 351000 ; 1993: 49000 ; 1994: 525000 ; 1995: 552000 ; 1996: 565000$ personnes, OCDE, Enquêtes « Emploi ", in B. MARCEL \& J. TAIEB, Le chômage aujourd'hui : Analyses et Perspectives, Paris : Nathan 1997, p. 18.

43. Bruno MARCEL \& Jacques TAIEB, op.cit., 1997.

44. Selon le BIT «le sous-emploi existe lorsque l'emploi d'une personne est insuffisant par rapport à des normes déterminées ou à un autre emploi possible compte tenu de la qualification professionnelle (formation ou expérience de travail) de l'intéressé » Claude THELOT, 'Le sous-emploi a doublé en 
quatre ans', Économie et Statistique, $\mathrm{n}^{\circ}$ 193-194, novembre-décembre, in B. MARCEL \& J. TAIEB, 1997, p. 20.

45. O. MAZEL, op. cit., 1999.

46. Béatrice MAJNONI D’INTIGNANO, 1993, La protection sociale, Paris : Éditions de Fallois, 1997, p.126.

47. J. Major : « A nous les emplois, à vous, les autres Européens, la protection sociale » J.P. FITOUSSI, op. cit., 1995, p. 248.

48. Le montant de l'Allocation de base est porté le $1^{\text {er }}$ janvier 1997 à 3100 francs contre 2800 francs.

49. Droits évalués en nombre de SMIC mensuels cumulés de 1979 à 1997 :

\begin{tabular}{|l|c|c|c|}
\hline \multicolumn{1}{|c|}{$\begin{array}{c}\text { Áges et } \\
\text { salaires }\end{array}$} & $\begin{array}{c}\text { Droits à } \\
\text { indemnisation en 1979 }\end{array}$ & $\begin{array}{c}\text { Droits à } \\
\text { indemnisation en 1997 }\end{array}$ & $\begin{array}{c}\text { Évolution } \\
\mathbf{1 9 7 9 - 1 9 9 7}\end{array}$ \\
\hline $\begin{array}{l}25 / 49 \text { ans } \\
1 \text { SMIC }\end{array}$ & 20,67 & 16,28 & $-21,2 \%$ \\
\hline $\begin{array}{l}25 / 49 \text { ans } \\
4 \text { SMIC }\end{array}$ & 43,67 & 52,73 & $+20,7 \%$ \\
\hline 50 ans I SMIC & 36,69 & 27,06 & $-26,2 \%$ \\
\hline 50 ans 4 SMIC & 80,35 & 84,25 & $+4,8 \%$ \\
\hline 55 ans I SMIC & 36,94 & 39,06 & $+5,7 \%$ \\
\hline 55 ans 4 SMIC & 81,19 & 131,70 & $+62,2 \%$ \\
\hline
\end{tabular}

IRES, 1999, in Christine DANIEL \& Carole TUCHSZIRER, L'Etat face aux chômeurs ; L'indemnisation du chômage de 1884 à nos jours, Paris : Flammarion, 1999, p. 314.

50. En 1998, 1100000 personnes bénéficiaient du RMI, O. MAZEL, op. cit., 1999, p. 127.

51. D'après une étude de Roger GODINO, G. AZNAR, op. cit., 1999, p. 259.

52. Rebondir, janvier 2002, $\mathrm{n}^{\circ} 102$ bis, p. 14 .

53. Deux brefs tableaux permettront peut-être de rendre compte de ces différences :

1. Les régimes d'assurance chômage : au $1^{\mathrm{er}}$ janvier 1992 et 1996 pour le Royaume-Uni et $1^{\text {er }}$ août 1993 pour la France :

\begin{tabular}{|c|c|c|c|}
\hline Pays & Conditions d'accès & $\begin{array}{c}\text { Taux de } \\
\text { remplacement }\end{array}$ & $\begin{array}{c}\text { Durée des } \\
\text { prestations }\end{array}$ \\
\hline Royaume-Uni & $\begin{array}{l}\text { Contributions égales à au moins } 50 \text { fois la limite } \\
\text { inférieure des gains hebdomadaires au cours des } \\
2 \text { dernières années }\end{array}$ & $\begin{array}{l}\text { Montant forfaitaire et } \\
\text { faible de l'ordre de } \\
25 \% \text { en moyenne du } \\
\text { salaire net antérieur }\end{array}$ & $\begin{array}{l}1 \text { an (6 mois } \\
\text { depuis } 1996)\end{array}$ \\
\hline France & $\begin{array}{l}4 \text { mois d'affiliation au cours des } 8 \text { demiers mois } \\
\text { pour recevoir une allocation } \\
6 \text { mois pour recevoir une allocation a taux plein }\end{array}$ & $\begin{array}{l}56 \% \text { en moyenne. } \\
57,4 \% \text { à } 75 \% \text { à taux } \\
\text { plein puis dégressivité } \\
\text { de } 8 \text { à } 17 \% \text { tous les } 4 \\
\text { mois }\end{array}$ & $\begin{array}{l}\text { De } 4 \text { à } 60 \text { mois suivants } \\
\text { la durée de l'affiliation et } \\
\text { de l'âge }\end{array}$ \\
\hline
\end{tabular}

2. Les dépenses publiques pour l'emploi et l'indemnisation des chômeurs en 1993 et 1995 :

\begin{tabular}{|l|c|c|c|c|}
\hline \multicolumn{1}{|c|}{ Pays } & $\begin{array}{c}\text { Dépenses en \% } \\
\text { du PIB en 1993 }\end{array}$ & $\begin{array}{c}\text { Dont } \\
\text { indemnisation }\end{array}$ & $\begin{array}{c}\text { Dont dépenses } \\
\text { pour I'emploi }\end{array}$ & $\begin{array}{c}\text { Dépenses en \% } \\
\text { du PIB en 1995 }\end{array}$ \\
\hline Royaume-Uni & 2,18 & 1,41 & 0,29 & 1,94 \\
\hline France & 3,31 & 1,57 & 1,3 & 3,1 \\
\hline
\end{tabular}

54. Catherine ANDRÉ, Dominique SICOT, Thomas SCHNEE \& Carole YEROCHEWSKI, Le chômage dans les pays industrialisés, Paris : Syros, 1994, p. 71.

55. Rebondir, janvier 2002, $\mathrm{n}^{\circ} 102$ bis.

56. Quelques indicateurs sur le chômage et l'emploi en 1998 : 


\begin{tabular}{|c|c|c|c|c|}
\hline Pays & & $\mathbf{1 5 / 2 4}$ ans & $\mathbf{2 5 / 5 4}$ ans & $\mathbf{5 5 / 6 4}$ ans \\
\hline France & Taux de chômage & $\mathbf{2 5 , 4} \%$ & $10,8 \%$ & $8,7 \%$ \\
& Taux d'activité & $\mathbf{2 8} \%$ & $86,2 \%$ & $36,1 \%$ \\
\hline Royaume-Uni & Taux de chômage & $12,3 \%$ & $5 \%$ & $5,3 \%$ \\
& Taux d'activité & $69,5 \%$ & $83,3 \%$ & $51 \%$ \\
\hline
\end{tabular}

D'après l'OCDE, Perspectives et Emploi, 1999, in O. MAZEL, 1999, p. 161.

57. Duncan GALLIE \& Susan ALM, 'Unemployment, Gender, and Attitudes to Work', in D. GALLIE \& S. PAUGAM, (eds.), Welfare Regimes and the Experience of Unemployment in Europe, Oxford: Oxford University Press, 2000, p. 113.

58. Nicolas BAVEREZ, Les trente piteuses, Paris : Flammarion, 1997.

59. N. BAVEREZ, op. cit., 1997, p. 52.

60. European Labour Force Survey, Employment Gazette, 1995.

61. O. MAZEL, op. cit., 1999, p. 188 \& 191.

62. Maria-Teresa PIGNONI, "Le chômage de longue durée " colloque Agir contre Le chômage de longue durée, Paris : Ministère du Travail, 1992.

63. Robert CASTEL, La métamorphose de la question sociale, Paris : Fayard, 1995, p. 717 ; Annick RENAUD-COULON \& Hervé SERYEX, Reprenons la Bastille: Le modèle de société au cœur de l'emploi, Paris : Village Mondial, 1999, p. 23.

64. A. RENAUD-COULON \& H. SERYEX, op. cit., 1999, p. 95.

65. European Community Household Panel, 1994, in D. GALLIE \& S. PAUGAM, op. cit., 2000, p.16.

66. European Community Household Panel, 1994, in D. GALLIE \& S. PAUGAM , op. cit., 2000, p. 51.

67. Hoover a fermé une filiale française pour s'établir en Écosse, où les montants des charges sociales étaient bien moindres. Cela paraît être une bonne solution pour les chefs d'entreprise mais est une catastrophe pour les employés car ils ne peuvent bénéficier de prestations sociales correspondant à leurs attentes, en particulier en cas de chômage ou de mise à la retraite. Les entreprises affirment qu'elles ne peuvent rester compétitives face à la concurrence asiatique, en oubliant qu'elles sont à la base de cette concurrence grâce aux délocalisations et aux transferts de technologie. Zola, dans Germinal, avait déjà dénoncé cet argument: dans le roman, un propriétaire de mine française opposait des mineurs français aux mineurs belges, qui acceptaient un salaire moindre pour maximiser ses profits.

\section{AUTEUR}

\section{JOËLLE HAREL}

Université de Paris Val-de Marne 